\title{
Cardio Protective Effects of Hydroalcholic Citrus Aurantium Extract on Myocardial Infarction Induced by Isoproterenol in Male Rats
}

\section{Abstract}

The citrus aurantium has traditionally been used by the people of Iran. The aim of this study was to evaluate the protective effects of hydroalcholic citrus aurantium extract on electrocardiographic, biochemical and pathological changes after myocardial infarction induced by isoproterenol in rats. 24 male Wistar rats were divided randomly into 4 groups: Control, extract, isoproterenol, and isoproterenol + pre-treatment with extract for two weeks. At the end of the experiment, ECG was recorded in lead II. Finally, blood samples were taken and cardiac marker enzymes such as creatine kinase, lactate dehydrogenase, aspartate aminotransferase, alanine aminotransferase, and alkaline phosphatase, along with oxidative stress markers such as malondialdehyde, superoxide dismutase, catalase, glutathione reductase,

Myocardial infarction induced by isoproterenol produced a significant increase in the heart rate, ST-segment elevation, decrease in R amplitude, and a significant increase in the level of cardiac marker enzymes: LDH, CK-MB, AST, ALT and ALP in serum. Also, isoproterenol significantly reduced SOD, CAT, GSH, GP and increased MDA activity, disturbance in lipid profile, and inflammatory process in cardiac myocytes. Pretreatment with citrus aurantium extract for two weeks significantly reduced the effect of isoproterenol on electrocardiographic parameters, cardiac biomarkers, oxidative stress indices, lipid profile and cardiac myocytes injuries.

Results indicated that citrus aurantium extract ameliorates the cardio-toxic effects of isoproterenol through reinforcement of antioxidant defense system and may be of value in treatment of myocardial infarction. Further studies are required to determine the precise mechanism of the therapeutic effect of the extract.

Keywords: Citrus aurantium; Isoproterenol; Myocardial infarction; Rat glutathione peroxidase, and lipid profile were determined.

\begin{tabular}{|c|}
\hline Research Article \\
\hline Volume 10 Issue 3 - 2017 \\
\hline \multirow{3}{*}{$\begin{array}{l}\text { Saeed Keshtkar }{ }^{1}, \text { Gholamreza Komeili }^{2 *} \text {, } \\
\text { Farshid Keshavarzi }{ }^{3} \text { and Mahdi Jahantigh }{ }^{4} \\
{ }^{1} \text { Student Research Committee, Faculty of Medicine, Zahedan } \\
\text { University of Medical Sciences, Iran }\end{array}$} \\
\hline \\
\hline \\
\hline $\begin{array}{l}\text { 2Department of Physiology, School of Medicine, Zahedan } \\
\text { University of Medical Sciences, Iran }\end{array}$ \\
\hline $\begin{array}{l}{ }^{3} \text { M.Sc. Student, Department of Clinical Biochemistry, Zahedan } \\
\text { University of Medical Sciences, Iran }\end{array}$ \\
\hline $\begin{array}{l}{ }^{4} \text { Pathology Department, School of Medicine, Zahedan } \\
\text { University of Medical Sciences, Iran }\end{array}$ \\
\hline $\begin{array}{l}\text { *Corresponding author: Gholamreza Komeili, Associate } \\
\text { professor in Physiology, Department of Physiology, School of } \\
\text { Medicine, Zahedan University of Medical Sciences, Zahedan, } \\
\text { Iran, Tel: +98 } 543 \text { 3235122; +989155412877; } \\
\text { Email: rkomeili@gmail.com; Komeili@Zaums.ac.ir }\end{array}$ \\
\hline $\begin{array}{l}\text { Received: December 19, } 2017 \text { | Published: December 27, } \\
2017\end{array}$ \\
\hline
\end{tabular}

\section{Introduction}

Although clinical care has been amended, public awareness elevated and health innovations are commonly used, Myocardial infarction [1] still remains the prominent cause of death worldwide [1]. According to the World Health Organization (WHO), it will be the most important cause of death in the world by the year 2020 [2]. MI is one of the usual forms of ischemic heart disease. The principle contributing factor in pathophysiologic condition of myocardial ischemia is disproportion in myocardial oxygen supply and demand. During myocardial ischemia, a wide range of cellular metabolic errors and structures damage mechanical dysfunction of myocardium by production of free radicals and reactive oxygen species leading to destruction of proteins, nucleic acids, carbohydrates as well as a variety of membrane lipids and subsequent cell deaths [3]. So, by considering the correlation with the pathogenesis of coronary artery diseases, oxidative stress accelerates generation of reactive oxygen species (ROS) and reduces antioxidant defenses [4].
Isoproterenol, a synthetic $\beta$-adrenoceptor agonist, has been found to induce MI in rats as an outcome of disorder in physiological balance between creation of free radicals and the antioxidant defense system [5]. It is the acute form of myocardial necrosis which sources cardiac malfunctions, increased lipid peroxidation, changed activities of cardiac enzymes and antioxidants [6]. Cardiac muscle necrosis induced by ISO, involves membrane permeability changes that produce loss of function and integrity of the cell membrane. Use of ISO drains the energy reserves of myocardial cells and complex biochemical and structural changes producing cell injury, which is preface to necrosis [7].

Citrus aurantium $\mathbf{L}$. is commonly dispersed in tropical and subtropical southeast areas of the world. The dried and immature peels of the citrus fruit are used in traditional herbal medicine [8]. This plant is a native and is commonly used in medicinal plants in Iran. In traditional Iranian medicine, the flowers of this plant were used for the treatment of neurological disorders such as hysteria, and seizures. In addition, this plant has been known as 
a sedative-hypnotic, appetizer and palpitation remover. Chemical compounds found in the plant are hydrocarbons, alcohols, some form of acetate, acids and phenols. Flavonoids are a class of polyphenols that are present in extracts of citrus aurantium flowers [9]. Citrus fruits are a common food source because of their nutrient, flavor, and intrinsic characteristics. They have long been the basis for usually used traditional medicines in some Asian countries [10]. Amid nutrients of citrus fruits, flavonoids have been more lately documented as having several benefits that contain antioxidant, antimicrobial, anti-inflammatory, and anti-cancer properties [11,12]. The flavonoids extracted from citrus fruit containing citrus aurantium $\boldsymbol{L}$. are mostly consisted of hesperidin, naringenin, and nobiletin. These flavonoids have been stated to have some properties that adjust the inflammatory response and halts carcinogenesis [13]

According to that, since the inflammatory process and antioxidant system involves MI induced by ISO, we hypothesized that this plant extract may have cardio protective effects. The purpose of this study was to explore the possible antioxidant effect of citrus aurantium flower extract and cardio protection against MI induced by ISO through electrocardiographic, myocardial marker injury, lipid profile changes as well as peroxidation of lipids, changes in antioxidant system and histopathological parameters.

\section{Materials and methods}

\section{Chemicals}

Isoproterenol hydrochloride was purchased from Sigma Aldrich Chemical Company, St. Louis, MO, USA. Lipid peroxidation product (MDA) and antioxidant assay kits (SOD, GSH, CAT, GP were bought from Zell Bio Company, Germany. Other biochemical substances were of analytical grade and purchased from ParsAzmoon Company, Iran.

\section{Animals}

All experiment and procedures defined in this study were approved by the local ethics committee of Zahedan University of Medical Sciences. Twenty four normal adult male Wistar albino rats weighting 200-250 g were selected and kept in single cages in standard laboratory settings (temperature $23 \pm 2^{\circ} \mathrm{C}, 12 \mathrm{~h}$ light and dark cycle, and 35-60\% humidity) for one week for adaptation $[14,15]$. Animals were fed with standard pellet diet and water ad libitum.

\section{Induction of experimental myocardial infarction}

ISO was dissolved in normal saline and injected into rats (150 $\mathrm{mg} / \mathrm{kg}$, s.c.) at $24 \mathrm{~h}$ interval for 2 days to experimental myocardial infarction induction [16].

\section{Experimental design}

After acclimatization, the animals were allocated randomly into 4 groups (6 in each group). These groups include control, group that received $600 \mathrm{mg} / \mathrm{kg}$ i.p. plant extract daily, group that received isoproterenol $150 \mathrm{mg} / \mathrm{kg}$, s.c. with $24 \mathrm{~h}$ interval on $13^{\text {th }}$ and $14^{\text {th }}$ days, treated group with plant extract $600 \mathrm{mg} / \mathrm{kg}$, i.p. daily and isoproterenol with $150 \mathrm{mg} / \mathrm{kg}$, s.c., with $24 \mathrm{~h}$ interval on $13^{\text {th }}$ and $14^{\text {th }}$ days. The volume of solution injected was same in all groups. Total duration of experiment was two weeks and all experiments started at 9 am every day.

\section{Electrocardiography}

At the end of the experimental duration, needle electrodes were implanted under the skin of animals in lead II position [15], under anesthesia with ketamine and xylazine $(80$ and $20 \mathrm{mg} / \mathrm{kg})$ intrapritoneally. ECG recordings obtained through computerized Power Lab system (AD Instruments, Australia) and analyzed with chart7 software.

\section{Biochemical analysis}

After recording the ECG, the animals were sacrificed and blood samples were taken. Samples were centrifuged at $4000 \mathrm{rpm}$ for ten minutes. Serum was taken and kept at $-70{ }^{\circ} \mathrm{C}$, and then CK-MB, LDH, ALP, AST, ALT, and lipid profile were measured using routine laboratory kits (Pars Azmoon, Tehran). Serum LDL-cholesterol was calculated by the Friedewald formula. MDA, SOD, GP ${ }_{x}, G S H$, and CAT were measured using Zell Bio kit (Zell Bio $\mathrm{GmbH}$, Deutschland) and ELISA method.

\section{Histopathology}

After sacrifice, the heart was quickly separated and washed immediately with saline and fixed in 10\% buffered paraformaldehyde solution. The fixed tissues were inserted in paraffin, sectioned at $5 \mu \mathrm{m}$ and stained with hematoxylin and eosin [17]. The samples were observed under light microscope, and then photomicrographs were taken. It should also be mentioned that grading of histopathological changes was classified by a blind pathologist as: low, mild (focal myocytes injury or multifocal deterioration with a mild degree of inflammation), moderate (myofibrillar degeneration or moderate inflammatory process) and severe (necrosis with extensive inflammatory process).

\section{Plant preparation and extraction}

Citrus aurantium flowers were taken from Shiraz, Iran, in 2016. The plant was recognized and authenticated by the botany department of Sistan and Baluchistan University. Twenty g of powdered flowers of citrus aurantium was placed in Soxhlet system with $70 \%$ ethanol for 8 hours. Then the extract was dehydrated in an incubator $\left(37^{\circ} \mathrm{C}\right)$ to give a final yield of about $10 \%$ hydroalcholic extract of plant. During the time of experiments, the extract was dissolved in saline and animals were forced-fed with above-mentioned doses [14].

\section{Statistical analysis}

Data is expressed as mean \pm SE. Statistical analysis was done by SPSS software version 17. Comparison of the data among groups was done using one-way analysis of variance followed by Tukey`s post hoc test, and non-parametric analysis (Kruskal-Wallis and Mann-Whitney U test) for histopathological examination. P $<0.05$ was considered statistically significant. 


\section{Results}

The effects of ISO and citrus aurantium extract treatment on pattern of ECG are depicted in Figure 1 and Table 1. Control and citrus aurantium treated rats showed normal pattern of ECG, whereas rats treated with ISO showed a significant increase in $\mathrm{S}$-T voltage, decrease in $\mathrm{R}$ amplitude as compared to control rats, suggestive of myocardial infarction. ISO treated rats likewise displayed the pathological $\mathrm{Q}$ wave, demonstrating transmural myocardial infarction induction. Also, a significant decrease in QRS and R-R interval and a significant increase in heart rate were detected in rats treated with ISO.

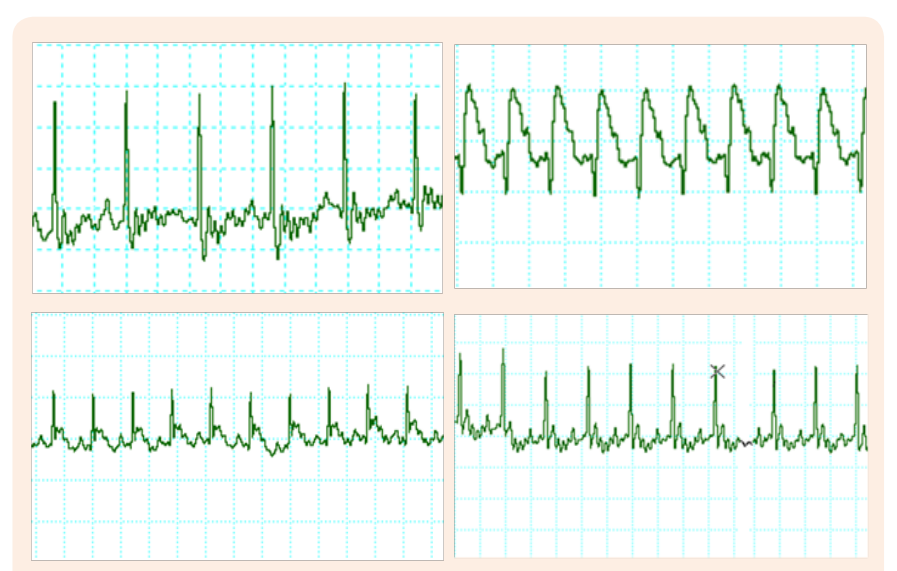

Figure 1: Electrocardiographic patterns in control (A), ISO (B), pretreated with citrus aurantium extract then treated with ISO (C) and citrus aurantium alone (D) groups.

Table 2 represents the effects of ISO and citrus aurantium extract treatment on cardiac marker enzymes such as CK-MB, LDH, ALP, AST and ALT. ISO treated rats showed an increased significantly in activities of these enzymes as compared to the control group. ISO treated animals that pre-treated with citrus aurantium extract exhibited significantly decrease the CK-MB, LDH, ALP, AST and ALT activities. No significant difference was detected in rats treated with the extract alone when compared to control rats.

The levels of MDA and GSH along with the activities of the enzymes $\mathrm{GP}_{\mathrm{x}}, \mathrm{SOD}$, and catalase in normal and experimental rats are listed in Table 3. The value of MDA, end product of lipid peroxidation and marker for oxidative stress, showed significantly increase in ISO treated rats, as compared to the control group. ISO treatment also caused in the significant decrease in the level of GSH, an endogenous antioxidant in comparison with normal control rats. Activities of glutathione-dependent antioxidant enzyme and antiperoxidative enzymes were significantly lowered in ISO treated rats as compared to the control group. Pre and co-treatment with citrus aurantium in MI induced by ISO rats significantly decreased the level of MDA as compared to rats treated with ISO alone. The pre-treatment of citrus aurantium for 14 days resulted in a significant rise in the level of GSH and activities of $\mathrm{GP}_{\mathrm{X}}$, SOD and catalase. Normal rats that receive citrus aurantium alone did not display any substantial alteration when compared with other normal rats, indicating that it does not per se have any adverse effects.

Table 4 lists the levels of total cholesterol, HDL, LDL and triglycerides in the serum of all groups of animals. Rats treated with ISO only exhibited a significant rise in these parameters with the levels of HDL-cholesterol being an exception where there was a significant decrease. Pre-treatment of citrus aurantium for 14 days beside with ISO administration on $13^{\text {th }}$ and $14^{\text {th }}$ days considerably reduced the levels of LDL and triglycerides with a following noteworthy increase in the levels of HDL-cholesterol as compared to ISO alone treated rats. No significant change in total cholesterol was observed when compared to ISO treated group. In the citrus aurantium alone group, there was no substantial alteration detected in serum lipoproteins and triglycerides levels in comparison to those of the control rats.

Table 1: Effect of citrus aurantium pretreatment on electrocardiographic parameters in ISO induced myocardial infarction in rats.

\begin{tabular}{|c|c|c|c|c|c|}
\hline Groups Parameters & Control & ISO & Citrus + ISO & Citrus & P value \\
\hline P wave (Sec) & $0.02 \pm 0.006$ & $0.02 \pm 0.001$ & $0.02 \pm 0.001$ & $0.02 \pm 0.001$ & 0.7 \\
\hline QRS complex(Sec) & $0.03 \pm 0.001$ & $0.05 \pm 0.001$ & $0.04 \pm 0.003$ & $0.03 \pm 0.002$ & 0.01 \\
\hline R amplitude ( $\mu v)$ & $383 \pm 15$ & $195 \pm 7$ & $287 \pm 16$ & $277 \pm 17$ & 0.001 \\
\hline QT interval (Sec) & $0.06 \pm 0.001$ & $0.07 \pm 0.003$ & $0.06 \pm 0.003$ & $0.05 \pm 0.001$ & 0.001 \\
\hline R-R interval (Sec) & $0.18 \pm 0.004$ & $0.15 \pm 0.01$ & $0.17 \pm 0.01$ & $0.18 \pm 0.004$ & 0.001 \\
\hline ST- segment ( $\mu v)$ & $50 \pm 4$ & $102 \pm 5$ & $47 \pm 8$ & $44 \pm 7$ & 0.001 \\
\hline Heart Rate (bpm) & $342 \pm 8$ & $412 \pm 30$ & $357 \pm 13$ & $335 \pm 10$ & 0.001 \\
\hline
\end{tabular}

All values are presented as Mean \pm S.E.M. for six rats in each group. 
Table 2: Effect of citrus aurantium pretreatment on cardiac marker enzymes in ISO induced myocardial infarction in rats.

\begin{tabular}{|c|c|c|c|c|c|}
\hline $\begin{array}{c}\text { Groups } \\
\text { Parameters }\end{array}$ & Control & ISO & Citrus + ISO & Citrus & P value \\
\hline CK-MB (IU/L) & $278.3 \pm 14.2$ & $646.8 \pm 70.1$ & $367.6 \pm 41.9$ & $277.5 \pm 14.7$ & 0.001 \\
\hline LDH (IU/L) & $1336 \pm 141.6$ & $2841 \pm 279.3$ & $1392.6 \pm 113.9$ & $1344.2 \pm 97.7$ & 0.001 \\
\hline ALP (IU/L) & $315 \pm 25.2$ & $421.3 \pm 25.8$ & $344.2 \pm 23.9$ & $281.7 \pm 23.9$ & 0.01 \\
\hline AST (U/L) & $155 \pm 6.9$ & $272.7 \pm 22.7$ & $159.7 \pm 17.4$ & $135.7 \pm 7.1$ & 0.001 \\
\hline ALT (U/L) & $43.8 \pm 3.8$ & $103.2 \pm 14.1$ & $55.7 \pm 2.4$ & $36.8 \pm 2.5$ & 0.001 \\
\hline
\end{tabular}

All values are presented as Mean \pm S.E.M. for six rats in each group.

Table 3: Effect of citrus aurantium pretreatment on lipid peroxidation, endogenous antioxidant and antioxidant enzymes in ISO induced myocardial infarction in rats

\begin{tabular}{|c|c|c|c|c|c|}
\hline $\begin{array}{c}\text { Groups } \\
\text { Parameters }\end{array}$ & Control & ISO & Citrus + ISO & Citrus & $P$ value \\
\hline $\mathrm{SOD}(\mathrm{U} / \mathrm{ml})$ & $12.7 \pm 0.96$ & $7.5 \pm 0.7$ & $11.2 \pm 0.65$ & $11.8 \pm 0.5$ & 0.001 \\
\hline CAT (U/ml) & $11.9 \pm 1.2$ & $6.3 \pm 0.3$ & $11.1 \pm 0.8$ & $11.6 \pm 1.2$ & 0.001 \\
\hline GSH (U/L) & $42 \pm 5.2$ & $27.5 \pm 3.2$ & $38.2 \pm 2.7$ & $49.5 \pm 3.4$ & 0.001 \\
\hline MDA (nmol) & $119.25 \pm 27.6$ & $316.5 \pm 34.1$ & $108.5 \pm 16.2$ & $145.5 \pm 22.9$ & 0.001 \\
\hline $\mathrm{GPX}(\mathrm{U} / \mathrm{ml})$ & $413.3 \pm 48.4$ & $224.2 \pm 52.5$ & $388.2 \pm 48.8$ & $435.6 \pm 19.9$ & 0.01 \\
\hline
\end{tabular}

All values are presented as Mean \pm S.E.M. for six rats in each group.

Table 4: Effect of citrus aurantium pretreatment on lipid profile in serum (mg/dl) of ISO induced myocardial infarction in rats.

\begin{tabular}{|c|c|c|c|c|c|}
\hline $\begin{array}{c}\text { Groups } \\
\text { Parameters }\end{array}$ & Control & ISO & Citrus + ISO & Citrus & P value \\
\hline Total cholesterol & $62.7 \pm 6.3$ & $92.7 \pm 8.7$ & $81.8 \pm 3.1$ & $50 \pm 2.9$ & 0.001 \\
\hline HDL-C & $31.3 \pm 1.5$ & $21.3 \pm 2.2$ & $28.5 \pm 0.9$ & $28.2 \pm 0.8$ & 0.01 \\
\hline LDL-C & $22.4 \pm 6.7$ & $58.7 \pm 11.7$ & $39.2 \pm 2.9$ & $14.6 \pm 1.7$ & 0.001 \\
\hline Triglyceride & $41.7 \pm 3.4$ & $71.5 \pm 8.8$ & $42.2 \pm 1.6$ & $53.5 \pm 5.8$ & 0.004 \\
\hline
\end{tabular}

All values are presented as Mean \pm S.E.M. for six rats in each group.

Figure 2 illustrates the histological photographs of heart tissues of all studied groups. Histopathological analysis of myocardial tissue achieved from normal control animals displayed clear integrity of membrane of myocardial cells. Normal untreated rats indicated typical cardiac tissues without any infarction and infiltration of inflammatory cells was not seen in this group. Histopathological results revealed that the ISO caused induction of MI in cardiac muscle. Tissues sample from myocardial infarction induced by ISO, exhibited extensive myocardial structure abnormality and subendocardial necrosis with capillary dilatation and leukocyte infiltration as compared to the control group. Prior administration of citrus aurantium showed reduced grade of infiltration of inflammatory cells and the appearance of myocardial cells was comparatively well conserved with no evidence of focal necrosis. Extract treated group rats displayed no change in morphology of heart tissue as compared to the normal control group.

\section{Discussion}

The pathophysiology mechanism of MI has not been fully revealed. Although catecholamines have regulatory effect on contraction and metabolism of myocardial muscle, these substances may be responsible for cell damages in the long term. The same situation can be observed in clinical situations such as angina, temporary myocardial hypoxia, acute inadequacy in coronary blood flow and subendocardial infarction. Isoproterenol, a potent synthetic catecholamine, can improve injuries like myocardial infarction when injected in animals. These lesions 
are apparently same to those of coagulative myocytolysis or myofibrillar deterioration. This is one of the findings during acute MI and unexpected death in man $[18,19]$.

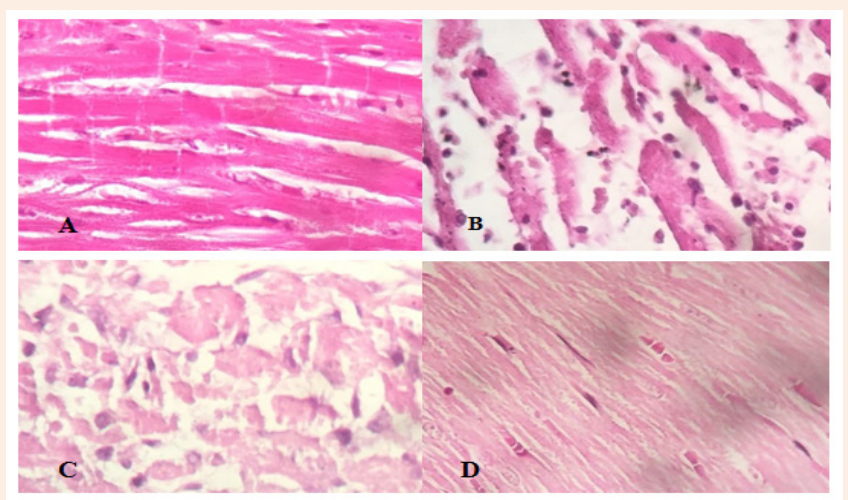

Figure 2: Effect of citrus aurantium pretreatment on histopathological changes \{(A) control, (B) ISO, (C) citrus aurantium + ISO, (D) citrus aurantium alone $\}$ in heart tissue in ISO induced myocardial infarction in rats (heart tissues were stained with hematoxylin and eosin and visualized under light microscope at 40X magnification).

Different mechanisms have been reported as responsible for induction of myocardial infarction by ISO. Previous reports recommended that coronary inadequacy, sarcoplasmic calcium excess, changed metabolic and electrolyte balancing system and oxidative stress are the main causative factors in catecholamine induced cardiac insufficiency [20]. Imbalance between oxygen delivery and request of myocytes after coronary hypotension and cardiac muscle hyperactivity (due to augmented heart rate and contractility) could cause myocardial damage [21]. Interaction of ISO with $\beta 1$ and $\beta 2$ adrenoceptors, activation of which causes to positive inotropic and chronotropic effects on heart, which produce relative ischemia due to myocardial hyperactivity and coronary hypotension [22].

ECG deliberated the single most important primary clinical exam for diagnosis of infarction and ischemia in cardiac muscle and. In our study, ISO administration causes pathological Q wave in rats, the most characteristic finding of transmural MI of the left ventricle. Administration of ISO also showed a decline in R-R interval, and increase in QRS time and heart rate. These changes could be due to the damage of cell membrane in injured cardiac muscle [23]. It has been exposed that a rise in heart rate is responsible for augmented oxygen consumption causing to enhanced necrosis of myocardial tissue [24]. ISO also increased ST-segment voltage and decreased R- wave voltage. This is favorable with the comments of earlier reports. ST-segment elevation reproduces the potential difference in the border between ischemic and non-ischemic regions and consequences in loss of cell membrane function whereas decrease in R-wave voltage might be due to the start of myocardial edema subsequent ISO treatment [25]. Citrus aurantium pre-treatment in ISO treated rats prohibited the pathological changes in the ECG, which suggests protective effect for its cell membrane.
Cardiac muscle contains different diagnostic markers of MI and when heart metabolism injured, it discharges its contents into the extracellular fluid [26]. Cytotoxic enzymes such as CKMB, LDH, AST, ALT and ALP, which serve as diagnostic markers, could be released from tissues damaged in the circulation due to permeable or disagreement of cell membrane and reflect the changes in plasma membrane integrity [27]. Our data confirmed significant increase in the levels of these enzymatic biomarkers of serum in ISO treated rats, which were in same direction with previous reports. Citrus aurantium pre-co-treatment caused in the dropped activity of the marker enzyme in serum. It validated that citrus aurantium could sustain cell membrane integrity, thereby limiting the leakage of these enzymes.

Free radical scavenging enzymes for example SOD, catalase and $\mathrm{GP}_{\mathrm{x}}$ are the first line of cellular protective system against oxidative stress, excluding reactive oxygen spices (ROS) such as superoxide and hydrogen peroxide. These enzymes inhibit the formation of more worsening hydroxyl radical. The additional line of guard includes non-enzymatic scavengers such as vitamin $\mathrm{C}$, vitamin E and sulphydryl containing compounds, which hunt remaining free radicals evading decomposition by the antioxidant enzymes [28]. The balance between antioxidant capacity and formation of free radicals is an essential process for the real elimination of oxidative stress in intracellular organelles. Though, in pathological situations similar to MI, the production of ROS can strongly interrupt this equilibrium with an augmented request of the antioxidant protection system [29].

Auto-oxidation induced by ISO can lead to production of massive quantity of ROS, which may attach any type of molecules such as polyunsaturated fatty acids in cell membrane forming peroxyl radicals, producing a chain reaction of lipid peroxidation [29]. This process is an essential pathogenic event in necrosis of myocardial tissue. MDA is a main end product of lipid peroxidation; raised MDA value may help to increased production of free radicals and/or reduced activities of antioxidant defense system [30]. In the current study, treatment with ISO caused in significant raise in peroxidation of lipids, represented as MDA value, which is in same direction with former reports [26,31]. Citrus aurantium pre-cotreatment displayed an important decrease in the value of serum MDA content which can be related to strong antioxidant capacity of extract against ISO auto-oxidation produced free radicals.

Activities of antiperoxidative enzymes (SOD and catalase) were declined considerably in serum of ISO treated rats. Superoxide dismutase, which catalysis the dismutation of two superoxide radicals to form hydrogen peroxide and molecular oxygen. Therefore, the $\mathrm{H}_{2} \mathrm{O}_{2}$ created is deactivated by either catalase or the GSH redox system containing of reduced glutathione as the cofactor for $\mathrm{GP}_{\mathrm{x}}$ and glutathione reductase [32]. Citrus aurantium pre-co-treatment exhibited a substantial increase in the level of serum SOD and catalase. These enzymes could not alter in the citrus aurantium animal group. In the current study, our data confirms a declined concentration of GSH and $\mathrm{GP}_{\mathrm{x}}$ in serum of ISO treated rats. GSH, a tripeptide, one of the greatest nonenzymatic antioxidant biomolecules, is existing in the body. GSH has a direct antioxidant role by reacting with superoxide radicals, 
peroxy radicals and singlet oxygen followed by the creation of oxidized GSH and other disulfides [33]. Diminished activity of these enzymes lead to formation of oxidants and make cardiac myocytes membranes more susceptible to oxidative injury. The current study revealed a significant increase in the value of GSH and activity of $\mathrm{GP}_{\mathrm{x}}$ in the serum of citrus aurantium pre-co-treated rats.

Lipids play a significant role in cardiovascular diseases. A considerable rise in the total cholesterol and triglycerides was detected in serum of ISO treated rats, which is in line with previous reports $[15,19]$. Rats treated with ISO also exhibited an increase in LDL fraction along with a decline in HDL cholesterol. These alterations could be credited to improved lipid biosynthesis by cardiac cyclic adenosine monophosphate [34]. A sturdy positive correlation has been document among the risk of emerging ischemic heart disease and serum LDL level, while a negative correlation has been stated with HDL-cholesterol [35]. The pre-co-treatment with citrus aurantium effectively restored the elevated triglycerides, LDL-cholesterol and total cholesterol and decreased HDL-cholesterol in the serum of this group.

Histopathological investigation of the cardiac muscle tissue in the control rat group represented clear integrity of the cardiac myocytes membrane and no inflammatory cell infiltration was detected. ISO treated rats group showed a cellular dissociative view with marked inflammatory infiltration. The pre-co-treatment with citrus aurantium extract showed reduced inflammatory cell infiltration and relatively normal view of myocardial cells. This confirmed cardio protective effect of hydroalcholic citrus aurantium extract on MI induced by ISO in rats.

\section{Conclusion}

In conclusion, we can say that the current study confirmed that subcutaneous high-dose injections of isoproterenol can produce MI in rats as evident from the release of myocytes damage markers in serum. Myocardial injuries are related with diminished antioxidant defense system in the heart's electrocardiographic and histopathological changes as well as release of inflammatory markers. Furthermore, provided with experimental evidence, the hydroalcholic citrus aurantium extract preserved antioxidant enzyme levels and enhanced cardiac performance. This conclusion may be a new way to understand the useful effects of citrus aurantium extract on cardio protection against myocardial injury, in which oxidative stress has long been identified to contribute to pathogenesis.

\section{Acknowledgement}

The authors are grateful to the Deputy of research and technology of Zahedan University of Medical Sciences for their financial support of research project no 7146. The authors acknowledge the department of Physiology and Biochemistry at Zahedan University of Medical Sciences for the support provided for this work.

\section{Conflict of Interest}

The authors declare that they have no conflict of interest.

\section{References}

1. Aronow WS (2006) Epidemiology, pathophysiology, prognosis, and treatment of systolic and diastolic heart failure. Cardiol Rev 14(3): 108-124.

2. Lopez AD, Murray CC (1988) The global burden of disease, 19902020. Nat Med 4(11): 1241-1243.

3. Bagatini MD, Martins CC, Battisti V, Gasparetto D, da Rosa CS, et al. (2011) Oxidative stress versus antioxidant defenses in patients with acute myocardial infarction. Heart Vessels 26(1): 55-63.

4. Lee BJ, Lin YC, Huang YC, Ko YW, Hsia S, et al. (2012) The relationship between coenzyme Q10, oxidative stress, and antioxidant enzymes activities and coronary artery disease. ScientificWorldJournal 2012: 792756.

5. Srivastava S, Chandrasekar B, Gu Y, Luo J, Hamid T, et al. (2007) Downregulation of CuZn-superoxide dismutase contributes to beta-adrenergic receptor-mediated oxidative stress in the heart. Cardiovasc Res 74(3): 445-455.

6. Peer PA, Trivedi PC, Nigade PB, Ghaisas MM, Deshpande AD (2008) Cardioprotective effect of Azadirachta indica A. Juss. on isoprenaline induced myocardial infarction in rats. Int J Cardiol 126(1): 123-126.

7. Todd GL, Cullan GE, Cullan GM (1980) Isoproterenol-induced myocardial necrosis and membrane permeability alterations in the isolated perfused rabbit heart. Exp Mol Pathol 33(1): 43-54.

8. Han MH, Lee WS, Lu JN, Kim G, Jung JM, et al. (2012) Citrus aurantium L. exhibits apoptotic effects on U937 human leukemia cells partly through inhibition of Akt. Int J Oncol 40(6): 2090-2096.

9. Huang YT, Wang GF, Chen CF, Chen CC, Hong CY, et al. (1995) Fructus aurantii reduced portal pressure in portal hypertensive rats. Life sciences 57(22): 2011-2020.

10. Deyhim F, Lopez E, Gonzalez J, Garcia M, Patil BS (2006) Citrus juice modulates antioxidant enzymes and lipid profiles in orchidectomized rats. J Med Food 9(3): 422-426.

11. Li Y, Fang H, Xu W (2007) Recent advance in the research of flavonoids as anticancer agents. Mini Rev Med Chem 7(7): 663-678.

12. Quilez A, Berenguer B, Gilardoni G, Souccar C, de Mendonca S, et al. (2010) Anti-secretory, anti-inflammatory and anti-Helicobacter pylori activities of several fractions isolated from Piper carpunya Ruiz \& Pav. J Ethnopharmacol 128(3): 583-589.

13. Nogata Y, Sakamoto K, Shiratsuchi H, Ishii T, Yano M, et al. (2006) Flavonoid composition of fruit tissues of citrus species. Bioscience, biotechnology, and biochemistry 70(1): 178-192.

14. Komeili G, Hashemi M, Bameri-Niafar M (2016) Evaluation of Antidiabetic and Antihyperlipidemic Effects of Peganum harmala Seeds in Diabetic Rats. Cholesterol 2016: 7389864.

15. Patel V, Upaganlawar A, Zalawadia R, Balaraman R (2010) Cardioprotective effect of melatonin against isoproterenol induced myocardial infarction in rats: A biochemical, electrocardiographic and histoarchitectural evaluation. Eur J Pharmacol 644(1-3): 160168.

16. Ribeiro DA, Buttros JB, Oshima CT, Bergamaschi CT, Campos RR (2009) Ascorbic acid prevents acute myocardial infarction induced by isoproterenol in rats: role of inducible nitric oxide synthase production. J Mol Histol 40(2): 99-105. 
17. Devi Sampath P, Vijayaraghavan K (2007) Cardioprotective effect of alpha-mangostin, a xanthone derivative from mangosteen on tissue defense system against isoproterenol-induced myocardial infarction in rats. J Biochem Mol Toxicol 21(6): 336-339.

18. Baroldi G (1974) Letter: Myocardial necrosis: the need for definition. J Mol Cell Cardiol 6(4): 401-402.

19. Variya BC, Patel SS, Trivedi JI, Gandhi HP, Rathod SP (2015) Comparative evaluation of HMG CoA reductase inhibitors in experimentally-induced myocardial necrosis: Biochemical, morphological and histological studies. Eur J Pharmacol 764: 283291.

20. Upaganlawar A, Balaraman R (2011) Cardioprotective Effects of Lagenaria siceraria Fruit Juice on Isoproterenol-induced Myocardial Infarction in Wistar Rats: A Biochemical and Histoarchitecture Study. J Young Pharm 3(4): 297-303.

21. Yeager JC, Whitehurst ME (1982) Verapamil prevents isoproterenolinduced cardiac failure in the rat. Life sci 30(3): 299-306.

22. Yeager JC, Iams SG (1981) The hemodynamics of isoproterenolinduced cardiac failure in the rat. Circulatory shock 8(2): 151-163.

23. Holland RP, Brooks H (1977) TQ-ST segment mapping: critical review and analysis of current concepts. Am J Cardiol 40(1): 110 129.

24. Rona G (1985) Catecholamine cardiotoxicity. J Mol Cell Cardiol 17(4): 291-306

25. Ramesh CV, Malarvannan P, Jayakumar R, Jayasundar S, Puvanakrishnan R (1988) Effect of a novel tetrapeptide derivative in a model of isoproterenol induced myocardial necrosis. Molecular and cellular biochemistry 187(1-2): 173-182.

26. Upaganlawar A, Gandhi C, Balaraman R (2009) Effect of green tea and vitamin $\mathrm{E}$ combination in isoproterenol induced myocardial infarction in rats. Plant Foods Hum Nutr 64(1): 75-80.
27. Farvin KH, Anandan R, Kumar SH, Shiny KS, Mathew S, et al. (2006) Cardioprotective effect of squalene on lipid profile in isoprenalineinduced myocardial infarction in rats. J Med Food 9(4): 531-536.

28. Sharma M, Kishore K, Gupta SK, Joshi S, Arya DS (2001) Cardioprotective potential of ocimum sanctum in isoproterenol induced myocardial infarction in rats. Mol Cell Biochem 225(1-): 75-83.

29. Singal PK, Kapur N, Dhillon KS, Beamish RE, Dhalla NS (1982) Role of free radicals in catecholamine-induced cardiomyopathy. Can J Physiol Pharmacol 60(11): 1390-1397

30. Zhou B, Wu LJ, Li LH, Tashiro S, Onodera S, et al. (2006) Silibinin protects against isoproterenol-induced rat cardiac myocyte injury through mitochondrial pathway after up-regulation of SIRT1. J Pharmacol Sci 102(4): 387-395.

31. Priscilla DH, Prince PS (2009) Cardioprotective effect of gallic acid on cardiac troponin-T, cardiac marker enzymes, lipid peroxidation products and antioxidants in experimentally induced myocardial infarction in Wistar rats. Chem Biol Interact 179(2-3): 118-1124.

32. Jayalakshmi R, Thirupurasundari CJ, Devaraj SN (2006) Pretreatment with alcoholic extract of Crataegus oxycantha (AEC) activates mitochondrial protection during isoproterenol - induced myocardia infarction in rats. Mol Cell Biochem 292(1-2): 59-67.

33. Meister A (1988) Glutathione metabolism and its selective modification. J Biol Chem 263(33): 17205-17208.

34. Ithayarasi AP, Devi CS (1997) Effect of alpha-tocopherol on lipid peroxidation in isoproterenol induced myocardial infarction in rats. Indian J Physiol Pharmacol 41(4): 369-376.

35. Karthikeyan K, Bai BR, Devaraj SN (2007) Efficacy of grape seed proanthocyanidins on serum and heart tissue lipids in rats subjected to isoproterenol-induced myocardial injury. Vascul Pharmacol 47(5-6): 295-301. 